\title{
VSTAR models of the hot Jupiter HD 189733b
}

\author{
Kimberly Bott ${ }^{1}$, Lucyna Kedziora-Chudczer ${ }^{1}$ and Jeremy Bailey ${ }^{1}$ \\ ${ }^{1}$ Department of Astrophysics, University of New South Wales, \\ Kensington, Sydney, Australia \\ email: k. bott@unsw.edu.au
}

\begin{abstract}
Past analysis of HD 189733b's atmosphere has been a cause for some debate, with conflicting findings regarding water and sodium abundances and the presence of a high altitude haze. We present our models of HD 189733b's atmospheric composition using VSTAR (Versatile Software for Transfer of Atmospheric Radiation). Since the effective temperature of the planet is expected to be approximately $5000 \mathrm{~K}$, newly available high-temperature spectral line lists were used.
\end{abstract}

Keywords. planets and satellites: individual: HD 189733b, planets and satellites: atmospheres, planets and satellites: composition, physical data and processes: radiative transfer

\section{Introduction}

The 1400 K Hot Jupiter, HD 189733b closely orbits (2.19 day) its K1.5 host star in a-most likely - tidally locked position, promising an extraordinary environment to study (Bouchy et al. 2005). Detection of water, CO2 and possibly methane have been reported in data from NICMOS (HST) and Spitzer (Swain et al. 2008, Tinetti et al. 2007). The broadband infrared data around 3.3 and $4 \mu \mathrm{m}$ suggests the presence of $\mathrm{CH} 4$ or $\mathrm{CO}$, although it is difficult to differentiate between the two currently. Conflicting models are perpetuated by the lack of data points in key absorption bands.

Here we compare our own models derived with VSTAR (Versatile Software for the Transfer of Atmospheric Radiation) to data from Charbonneau et al. (2008), Grillmair et al. (2008), and Swain et al. (2009), as well as to other models, particularly Lee et al. (2011), to derive a better understanding of how these effects come into play and to further establish the capability of VSTAR (Bailey \& Kedziora 2012). VSTAR's plane-parallel radiative transfer solution utilizes line-by-line absorption and particle scattering.

We ultimately aim to develop a model that can fit a wide range of data including transmission spectroscopy (e.g., Pont 2013), high resolution cross correlation spectroscopy (e.g., de Kok et al. 2013) and polarization (e.g., Berdyugina et al. 2008, 2011; Wiktorowicz 2009). At present we consider only the spectrum derived from secondary eclipse observations.

\section{The Lee 2011 Model}

We began with attempting to emulate a relatively straightforward model from Lee et al. (2011) which included only four molecules (CH4, H2O, CO and CO2) at a constant abundance over all heights and a retrieved Pressure-Temperature profile, wellsituated, mid-range of other suggested P-T profiles (e.g., Madhusudan \& Seager 2009). Although significance testing is not yet complete, our model, with abundances predicted by 
equilibrium chemistry, does appear to be a better fit than the model emulating Lee et al. (2011)'s constant ratios with height for the Spitzer data.

\section{Variables}

Four P-T profiles were compared from Lee et al. (2011) and Madhusudan \& Seager (2009). None of the profiles include inversion, as the dayside of HD 189733b appears to lack one Fortney et al. (2007), Charbonneau et al. (2008). Cooler T-P profiles appear to give better fits, particularly for shorter wavelengths. Further modelling and significance testing is needed to determine the scale of the effects.

Water features in the spectrum of HD $189733 \mathrm{~b}$ are seen in the data points from NICMOS around 2 microns, however these bands have some overlap with $\mathrm{CO}, \mathrm{CO} 2$ and $\mathrm{CH} 4$ Grillmair et al. (2008). A more convincing feature is the gap between vibrational modes of water vapour near 6.5 microns seen previously in other models and in our own. The broad absorption bands of water are clearly visible in the relatively well observed 5 to $10 \mu \mathrm{m}$ Spitzer data. The steep slope produced by water absorption around $3.5 \mu \mathrm{m}$ could be better constrained with further observational data.

The C/O level of HD189733b was estimated to be slightly more than solar (0.54) at 0.65 by Lee et al. (2011). Previously, Swain et al. (2009) placed the value broadly between 0.5 and 1.0. For most of our models $\mathrm{C} / \mathrm{O}$ of 0.65 was used and provided a good fit for the majority of the points.

\section{Results and Discussion}

Water absorption, while it seems to be needed for a good fit can have overlap with other bands. The predictable shape of the peaks between bands make it seem unlikely that an amalgamation of other nearby absorption bands is the culprit, but further analysis is needed to determine this.

The models presented here do not include scattering particles. A scattering haze is likely to be important to modelling the transmission spectroscopy (e.g. Pont et al. 2013) which appears to be consistent with the presence of Rayleigh scattering, probably due to small dust grains.

\section{References}

Bailey, J. \& Kedziora, L. 2012, MNRAS, 419, 1913-1929

Berdyugina, S. V., Berdyugin, A. V., Fluri, D. M., \& Piirola, V. 2008, ApJL, 673, L83-L86

Bouchy, F., Udry, S., Mayor, M., et al. 1995, A\&A, 444, L15-L19

Charbonneau, D., Knutson, H. A., Barman, et al. 2008, ApJ, 686, 1341-1348

de Kok, R. J., Brogi, M., Snellen, I. A.G., Birkby, J., Albrecht, S., \& de Mooij, E. J.W. 2013, $A \& A, 554, \mathrm{~A} 82$

Fortney, J. J., Lodders, K., Marley, M. S., \& Freedman, R. S. 2007, ApJ, 678, 1419-1435

Grillmair, C. J., Burrows, A., Charbonneau, et al. 2008, Nature, 456, 767-769

Madhusudan, N. \& Seager, S. 2009, ApJ, 707, 24

Lee, J. M., Fletcher, L. N., \& Irwin, P. G. J. 2011, MNRAS, 420, 170-182

Pont, F., Sing, D. K., Gibson, N. P., Aigrain, S, Henry, G., \& Husnoo, N. 2013, MNRAS, 432, $2917-2944$

Swain, M. R., Vasisht, G., Tinetti, G., et al. 2009, ApJL, 690, L114-L117

Swain, M. R., Tinetti, G., Vasisht, G., et al. 2009, ApJ, 704, 1616-1621

Tinetti, G., Vidal-Madjar, A., Liang, M.-C., et al. 2007, Nature, 448, 169-171

Wiktorowicz, S. J. 2009, ApJ, 696, 1116-1124 